\title{
PARACONDUCTIVITY OF \\ THREE-DIMENSIONAL AMORPHOUS SUPERCONDUCTORS:-E VIDENCE FOR A SHORT-WAVELENGTH CUTOFF IN THE FLUCTUATION SPECTRUM
}

$$
\text { To: } M P R
$$

MASTER

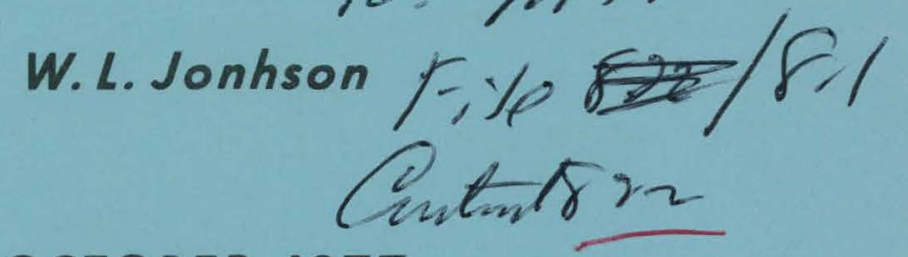

OCTOBER 1977

A REPORT ON RESEARCH CONDUCTED

UNDER CONTRACT FOR THE

U.S. ENERGY RESEARCH AND DEVELOPMENT

ADMINISTRATION

W. M. KECK LABORATORY OF

ENGINEERING MATERIALS

$$
\text { ACOZ-768R00822 }
$$

CALIFORNIA INSTITUTE OF TECHNOLOGY

PASADENA 


\section{DISCLAIMER}

This report was prepared as an account of work sponsored by an agency of the United States Government. Neither the United States Government nor any agency Thereof, nor any of their employees, makes any warranty, express or implied, or assumes any legal liability or responsibility for the accuracy, completeness, or usefulness of any information, apparatus, product, or process disclosed, or represents that its use would not infringe privately owned rights. Reference herein to any specific commercial product, process, or service by trade name, trademark, manufacturer, or otherwise does not necessarily constitute or imply its endorsement, recommendation, or favoring by the United States Government or any agency thereof. The views and opinions of authors expressed herein do not necessarily state or reflect those of the United States Government or any agency thereof. 


\section{DISCLAIMER}

Portions of this document may be illegible in electronic image products. Images are produced from the best available original document. 


\title{
California Institute of Technology
}

W. M. Keck Laboratory of Engineering Materials

\author{
PARACONDUCTIVITY OF THREE-DIMENSIONAL AMOR PHOUS \\ SUPERCONDUCTORS: /EVIDENCE FOR A SHORT-WA VELENGTH \\ CUTOFF IN THE FLUCTUATION SPECTRUM
}

by

W. L. Johns on

in collaboration with C. C. Tsuei and P. Chaudhari

IBM T. J. Watson Research Center

Yorktown Heights, New York 10598

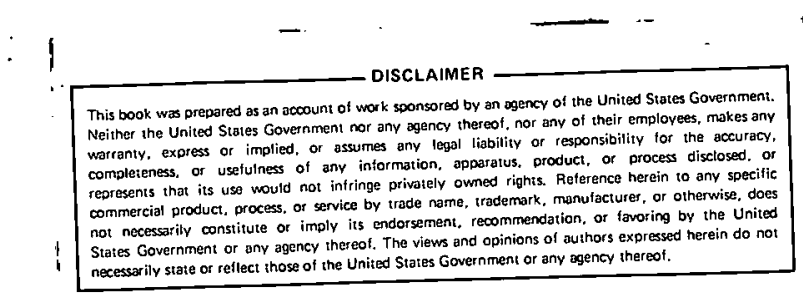

Energy Research and Development Agency Report No. 86, under Contract No. EY -76-C-03-0822.

Professor Pol Duwez, principal investigator.

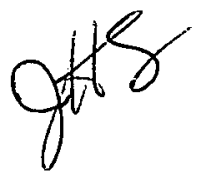




\section{NOTICE}

This report was prepared as an account of work sponsored by the United States Government. Neither the United States nor the Energy Research and Development Administration, nor any of their employees, nor any of their contractors, subcontractors, or their employees, makes any warranty, express or implied, or assumes any legal liability or responsibility for the accuracy, completeness or usefulness of any information, apparatus, product or process disclosed, or represents that its use would not infringe privately-owned rights. 


\begin{abstract}
Measurements of the temperature dependence and magnetic field dependence of the paraconductivity of a three dimensional amorphous superconductor are presented. The data are analyzed in terms of several current theories and are found to give good agreement for low fields and temperatures near $T_{c} \cdot$ The paraconductivity falls well below predicted theoretical values in the high temperature and high field limits. This is attributed to the reduced role of high wavevector contributions to the paraconductivity: It is shown that the introduction of a short wavelength cutoff in the theoretical fluctuation spectrum provides a phenomelogical account of the discrepancy between theory and experiment.
\end{abstract}




\section{INTRODUCTION}

Over the past ten years, there has been considerable interest in the study of the effects of thermal fluctuations on the properties of superconductors. In particular, the paraconductivity has been studied experimentally ${ }^{1,2,3,4}$ in the case of thin superconducting films, and also in bulk type II superconductors. 5 Much theoretical work has been devoted to predicting the temperature and magnetic field dependences of the paraconductivity beginning with the early work of Aslamazov and Larkin $^{6}(\mathrm{~A}-\mathrm{L})$ and followed by several others. $7,8,9,10$ Recently, two of the authors reported measurements of the temperature dependences of the paraconductivity of bulk amorphous superconductors. 11 Fluctuation effects in bulk amorphous superconductors a re strongly enhanced by the short electron mean free path and can be measured over a broad range of reduced temperature owing to the nearly temperature independent normal state conductivity. A universal temperature dependence of the. paraconductivity was found based on several samples studied. These results were found to be in good accord with theory for temperatures close to $T_{c}$ but exhibit strong deviations from theory at higher temperature.

In the present paper, we present new measurements of both the temperature and magnetic field dependence of the paraconductivity of an amorphous superconductor. The temperature dependence follows that previously reported for other amorphous superconductors. Both the temperature and field dependences show strong deviations from theoretical predictions at high temperature and high field. The paraconductivity falls much more rapidly than predicted in these limits. It 
is proposed that this behavior arises from the strongly.reduced role of short wavelength fluctuations. The effect can be taken into account by introducing a short wavelength cutoff in the fluctuation spectrum. The cutoff can be determined by fitting the data and is found to within experimental uncertainty to be the reciprocal of the zero temperature coherence length $\xi(0)$. A detailed theory of this effect would have to take into account the internal structure of a bound pair, and in particular the fact that in an amorphous superconductor one may in certain situations approach the limit of isolated pairs owing to the fact that $\xi(0)$ is extremely small. The former effects have been taken into account previously in calculations of the diamagnetic susceptibility of superconductors above $T_{c^{\cdot}}{ }^{12,13,14}$ The present results for the fluctuation conductivity show very similar features when compared with experimental results for the diamagnetic susceptibility. ${ }^{15}$ Taken together, these results imply that the theory of fluctuation phenomena in superconductors is with suitable extension capable of providing a consistent account of available experimental data.

\section{EXPERIMENTAL}

Measurements of the fluctuation conductivity were performed on several specimens of amorphous $\mathrm{Zr}_{75} \mathrm{Rh}_{25}$ prepared by rapid quenching from the liquid state. ${ }^{16}$ Measurements of $T_{c}$ were found to give slightly higher $\left(\mathrm{T}_{\mathrm{c}} \cong 4.5^{\circ} \mathrm{K}\right.$ ) values than those previously reported for this amorphous system. 17 In addition, it was found that the width of the superconducting transition was much less $\left(\Delta \mathrm{T}_{\mathrm{c}}=7 \times 10^{-3} \mathrm{o}_{\mathrm{K}}\right.$ as measured by the $10 \%$ to $90 \%$ points on the resistance curve) than that 
roportcd in ief. 17. This is attributed to differences in the two quenching apparatus. The alloys were prepared by induction melting of the constitutents on a silver boat under an argon atmosphere and slowly cooled to ensure homogeneity. The ingots were subsequently broken into small fragments which were then used to quench foils which have a thickness of $\sim 45 \mu \mathrm{m}$ and an area of several $\mathrm{cm}^{2}$. The structure was checked by $x$-ray diffraction scans using a Norelco diffractometer ( $\mathrm{Cu}-\mathrm{K} \alpha$ radiation). All foils showing evidence of crystalline phase precipitation were rejected for use in this study.

The sample resistivities were measured for temperatures ranging from $1.9 \mathrm{~K}$ to $10 \mathrm{~K}$ and magnetic fields ranging up to $75 \mathrm{KG}$ produced by a superconducting solenoid. The current densities used in the measurement were $\lesssim: 10 \mathrm{Amps} / \mathrm{cm}^{2}$. The sample is located in an exchange gas container the temperature of which can be stabilized to better than $1 \mathrm{mK}$. The temperature is measured using a carbon resistor with the calibration checked against the vapor pressure of He I during each run. The absolute accuracy of the temperature measurement is $\pm 50 \mathrm{mK}$ while the relative accuracy (near $T_{c}$ ) is $\pm 1 \mathrm{mK}$. The specimens used in the measurements were long strips with width $\sim 1 \mathrm{~mm}$ and length $\sim 1-2 \mathrm{~cm}$. The sample resistance was determined to an accuracy of better than 1 part in $10^{4}$. The error in the absolute resistivity is $\pm 10 \%$ due mainly to uncertainty in the sample geometry. The normal state resistivity was also measured up to room temperature for reference in a separate experimental station.

Finally, in order to establish the generality of the field dependence of the fluctuation conductivity, measurements were carried out on the La ${ }_{78} \mathrm{Au}_{22}$ amorphous alloy previously studied in ref. 11 and compared 
to those obtained or amorphnns $\mathrm{Zr}_{75} \mathrm{Rh}_{25^{\circ}}$

\section{EXPERIMENTAL RESULTS AND COMPARISON WITH THEORY}

To begin, a summary of relevant parameters characterizing the superconducting state of amorphous $\mathrm{Zr}_{75} \mathrm{Rh}_{25}$ is given for reference. The transition temperature in zero field $T_{\text {co }}$ varied by $\pm 0.05 \mathrm{~K}$ for the several samples studied. The value $T_{\text {co }}=4.562 \mathrm{~K}$ given in the table refers to the most homogeneous sample and is defined as the highest temperature for which the measured sample resistance $R=0$. The width of the superconducting transition for this sample was. $\Delta T_{c o}=7 \mathrm{mK}$ defined by the $10 \%$ and $90 \%$ points on the resistive transition curve. The resistive transition is shown in Fig. 1. This transition is somewhat sharper than those observed in the most homogeneous samples used in the study of ref. 11 permitting analysis of the fluctuation conductivity extending closer to $\mathrm{T}_{c}$. The temperature dependence of the upper critical field of the sample $H_{c 2}(t)\left(t=T / T_{c o}\right)$ was measured for reference in comparing the field dependence of the fluctuation conductivity to that predicted by theory. The results are shown in Fig. 1 and compared to the predictions of the Ma:i theory ${ }^{18}$ (with no paramagnetic limiting or spin-orbit effects taken into account) for $\mathrm{H}_{c 2}(t)$. Effects of surface superconductivity were also observed. Between $\mathrm{H}_{\mathrm{c} 2}(\mathrm{t})$ and $\mathrm{H}_{\mathrm{c} 3}(\mathrm{t})$; surface superconductivity persists in spite of the absence of a stable bulk superconducting state. In the analysis of the fluctuation conductivity, care was taken to on?y compare the fluctuation conductivity with theoretical predictions only for $\mathrm{H}>\mathrm{H}_{\mathrm{c} 3}(t)$ in order to avoid confusing it with the effects of surface superconductivity. This is discussed 


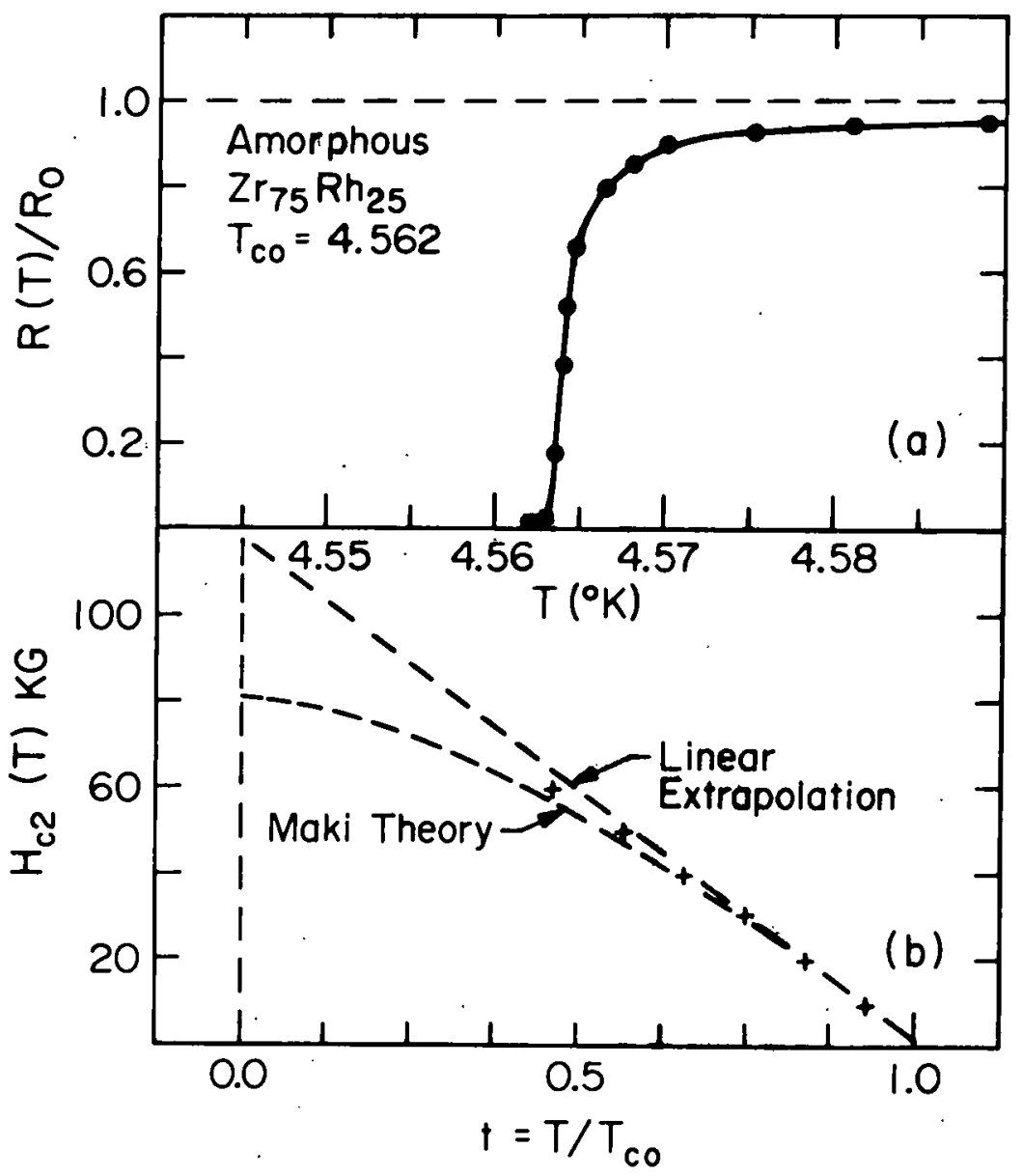

Fig. 1. (a) Normalized resistance vs. temperature and

(b) the upper critical field $\mathrm{H}_{\mathrm{c} 2}$ vs. reduced temperature $t=T / T_{c}$ for amorphous $\mathrm{Zr}_{75} \mathrm{Rh}_{25}$. The data for $\mathrm{H}_{\mathrm{c} 2}(\mathrm{t})$ are compared with the predictions of Maki (ref. 18) assuming no paramagnetic limiting and no spin-orbit effect. 
more fully later.

The measurement of the fluctuation conductivity was carried out in two ways. In the first, the field is held constant and the sample resistance measured as a function of temperature. In the second, the temperature is fixed and the field is varied from $H=0$ up to $H=75 \mathrm{KG}$. The results of the first set of measurements are shown in Fig. 2 . It is clear that the width $\Delta \mathrm{T}_{c}$ of the superconducting transition increases with increasing field. This effect can to a large extent be attributed to surface superconductivity as previously mentioned. For $\mathrm{H}=0$, the dependence of the excess conductivity $\sigma^{\prime}$ on temperature is illustrated by plotting $\ln \left(\sigma^{\prime} / \sigma_{o}\right)$ vs. en t* (where $t *=\left(T-T_{c}\right) / T T_{c}$ and $\sigma_{0}=$ normal state conductivity at $3 \mathrm{~T}_{c}$ ). This is shown in Fig. 3 along with previous data for amorphous La $78 \mathrm{Au}_{22}$ and compared with the prediction of the A-L theory. As discussed in ref. Il, the MakiThompson ${ }^{7}$ contribution to $\sigma^{\prime}$ is small: For amorphous $\mathrm{Zr}_{75} \mathrm{Rh}_{25}$, the Maki terms make a negligible contribution as evidenced by the good agreement between the magnitude of $\sigma^{\prime}$ predicted by the $A-L$ theory and experimental data. It should be noted that the re are two additional curves in Fig. 3 which are based on a modified $A-L$ theory which includes a short wave length cutoff in the fluctuation spectrum. This will be described in detail in the following section. Comparing the experimental results with the prediction of $A-L$ shows that the temperature dependence of $\sigma^{\prime}$ is well described near $T_{c}(-6 \lesssim \ell n t * \lesssim-3)$ whereas the data fall below the A-L predictions for higher temperature. The main difference as compared to the results of ref. 11 is the extended range of agreement between experiment and theory for $t * 0$. This as pre viously noted is attributed to improved sample homogeneity. 


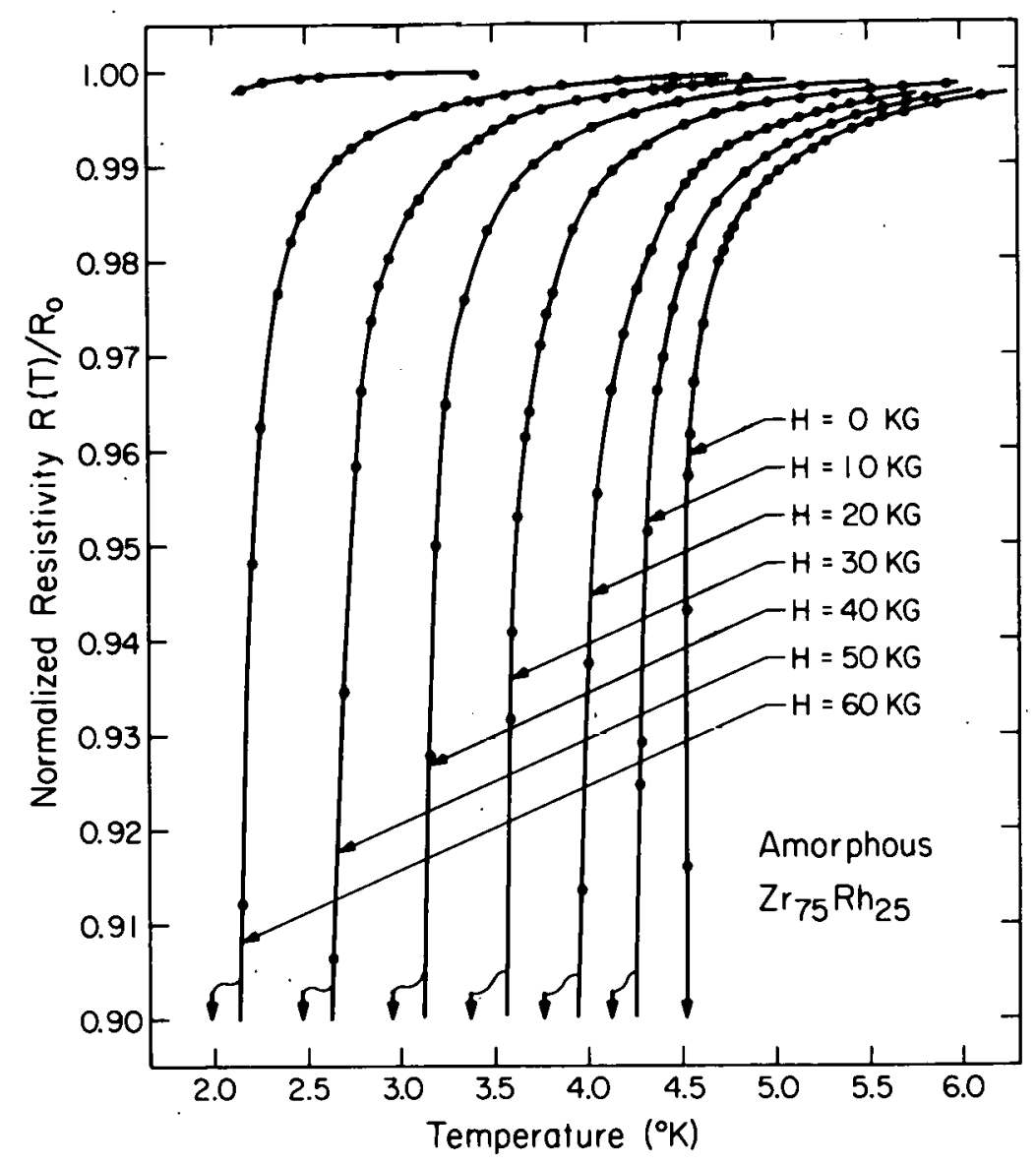

Fig. 2. Normalized resistance vs, temperature with various applied magnetic fields. The arrow in the lower part of the graph indicates the temperature for which $R=0$ in each applied field. 


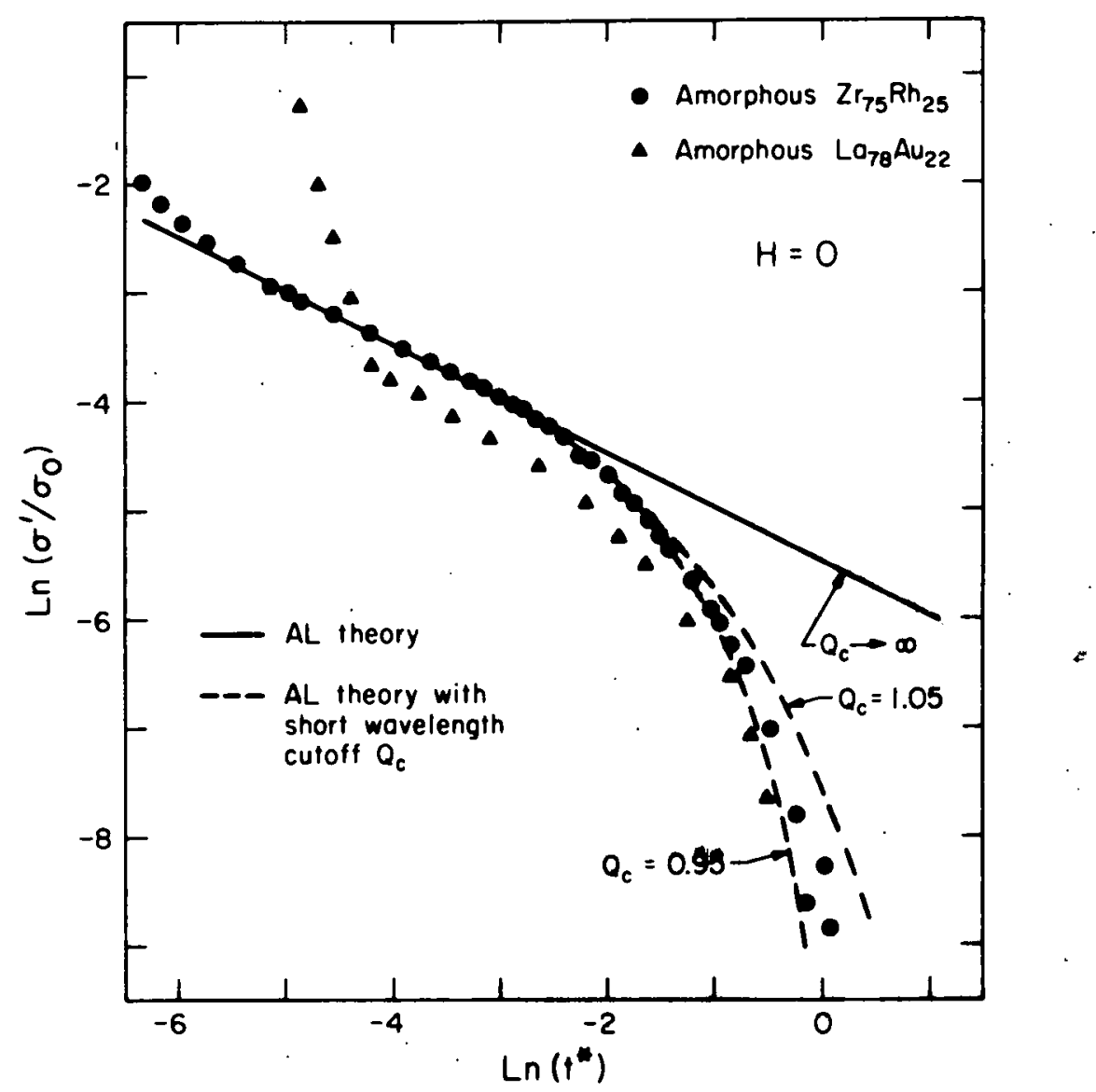

Fig. 3. The temperature dependence of the fluctuation conductivity $\left(\ell n \sigma^{\prime}\right.$ vs. $\ell$ n $\left.t^{*}\right)$ for $H=0$. The solid line is the prediction of the AL theory for amorphous $\mathrm{Zr}_{75} \mathrm{Rh}_{25}$. The dashed lines refer to the modified AL theory with a short wavelength cutoff. 
The resistive transition as a function of applied field was measured at constant temperature with the applied field perpendicular to the direction of current flow. The results are shown in Fig. 4. These results can be compared with the predictions of Maki ${ }^{7}$ or with those of Usadel. ${ }^{8}$ From equation (16) of ref. $7(\mathrm{c})$, the Maki result is expressed in the following form for the case of $H$ applied perpendicular to the direction of current flow.

$$
\left.\sigma_{1}^{\prime}=e^{2} / \pi(2 \pi)^{2}\left(4 \pi K_{B} T\right) / \pi D\right)[x(H, T)]^{-\frac{1}{2}}
$$

in cgs units with $\mathrm{e}^{\prime}=$ electron charge, $\mathrm{D}=$ electronic diffusivity and $\mathrm{X}$ a function of field and temperature given by

$$
X(H, T)=\frac{\ln \left(T / T_{c o}\right)+\psi\left[\frac{1}{2}+\left(e D H / 2 \pi K_{B} T\right)\right]-\psi\left(\frac{1}{2}\right)}{\psi(1)\left[\frac{1}{2}+\left(e D H / 2 \pi K_{B} T\right)\right]}
$$

where $T_{c o}$ is the transition temperature in zero field and $\psi$ and $\psi$ are respectively the digamma and trigamma functions. The electronic diffusivity is experimentally determine from the gradient of the upper critical field $\left[\mathrm{dH}_{\mathrm{c} 2}(\mathrm{~T}) / \mathrm{dT}\right]_{\mathrm{T}=\mathrm{T}_{\mathrm{co}}}$. The remaining parameters are all fixed leaving no free parameters. Equation (1) was evaluated as a function of field at several fixed temperatures and then compared with the experimental data for $\mathrm{Zr}_{75^{\mathrm{Rh}}} 5^{\circ}$. The data and theoretical pre dictions were compared by plotting $2 n \sigma^{\prime}$ as a function of $\ln \left(\mathrm{H}-\mathrm{H}_{\mathrm{c} 2}(\mathrm{t})\right) / \mathrm{H}_{\mathrm{c} 2}(\mathrm{t})=\operatorname{lnh}$. For the experimental data, the reduced field $H_{i}$ is defined in terms of the experimentally measured $\mathrm{H}_{c 2}(\mathrm{t})$ at various fixed temperatures. Disagreement between the theoretical 


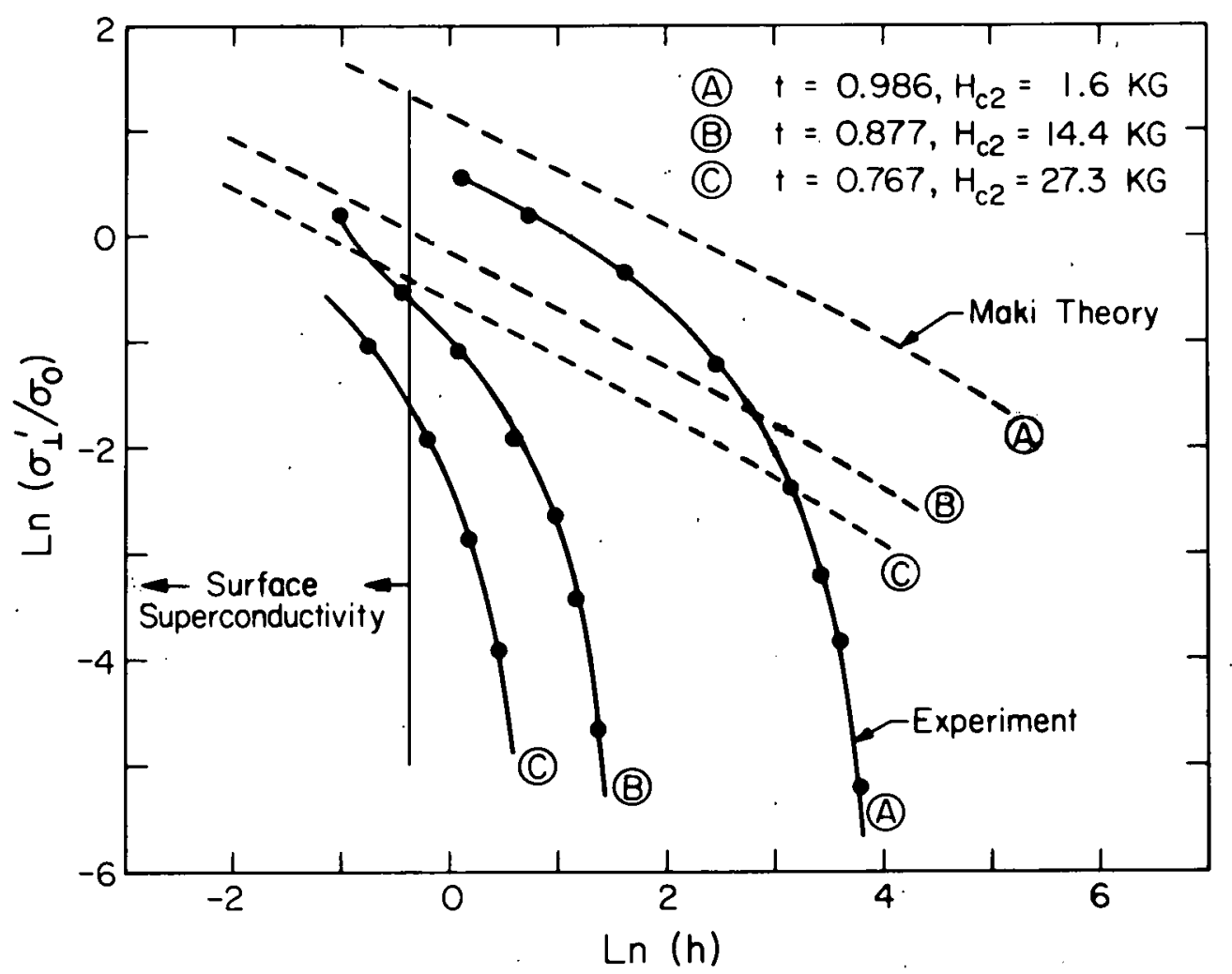

Fig. 4. Magnetic field dependence of the fluctuation conductivity $\sigma^{\prime}$ at several reduced temperatures. Results are compared to the Maki theory (dashed lines) as computed from equation (1) of the text. 
(Maki) $\mathrm{H}_{\mathrm{c} 2}(\mathrm{t})$ curve (Fig. 1) with no paramagnetic us spin orbit effects included and the experimental curve $\mathrm{H}_{\mathrm{c} 2}(\mathrm{t})$ is taken into account by this procedure in a manner noted in ref. 5. In analyzing this data, care was taken to avoid confusing the effects of fluctuation conductivity with those of surface superconductivity. Theoretically, it is known that $\mathrm{H}_{\mathrm{c} 3}(\mathrm{t}) \lesssim 1.69 \mathrm{H}_{\mathrm{c} 2}(\mathrm{t})$. By plotting the experimental data for low temperature where $\mathrm{H}_{\mathrm{c} 2}$ is fairly large, one can observe a clear break in the $\sigma$ vs. $\mathrm{H}$ curves typically for $\mathrm{H} \simeq 1.5 \mathrm{H}_{\mathrm{c} 2}$. This was interpreted as surface superconductivity. In comparing the experimental field dependence of $\sigma^{\prime}$. with theory, such regions were avoided. In Fig. 4 regions of possible surface superconductivity are indicated by a vertical line and arrows. In analogy with Fig. 3, it is found that at low fields $\mathrm{H}<\ll \mathrm{H}_{\mathrm{c} 2}(0)$ the theory and experiment are in good agreement whereas for $\mathrm{H} \lesssim \mathrm{H}_{\mathrm{c} 2}(0)$ the experimental values of $\sigma_{1}^{\prime}$ fall considerably below those predicted by theory. It is worthwhile to point out that the deviations from theory occur at roughly the same absolute field $H$ for all fixed temperatures. Since the reduced field $h$ is defined in terms of $\mathrm{H}_{\mathrm{C} 2}(\mathrm{t})$, the deviations occur at different values of $h$.

The field dependence of the fluctuation conductivity of amorphous La ${ }_{78} \mathrm{Au}_{22}$ was also measured. A plot similar to that in Fig. 4 shows that the experimental data follow very closely the behavior observed for amorphous $\mathrm{Zr}_{75^{\mathrm{Rh}}} 5^{\circ}$. This result is taken to confirm that the field dependence observed is general just as the temperature dependence found in ref. 11 appears to be general for three dimensional amorphous materials.

The temperature dependence of $\sigma^{\prime}$ in a constant nonzero applied field can be compared with theory by plotting $\ln \left(\sigma^{\prime} / \sigma_{0}\right)$ as a function of 
$\ln \left[\left(T-T_{c}(H)\right) / T_{c}(H)\right]=\ln t^{*}(H)$ wliere ' ${ }_{c}(H)$ is transition temperature of the superconductor in the field $H$. The value of $T_{c}(H)$ was determined from experimental data using the $R=0$ criteria. It was necessary to consider the flux-flow conductivity in defining $T_{c}(H)$. A small, but detectable resistance which is particularly observable in high fields was found to be present for $T<T_{c}(H)$. This small resistance is of order $10^{-3}-10^{-4}$ of the normal state resistance and has been shown to be associated with flux-flow. This resistance was ignored in defining $T_{c}(H)$ which was determining by linear extrapolation of the $R$ vs. $T$ curve (with $\left(R / R_{0}\right)>10 \%$ ) to $R=0$. The results of above comparison are shown in Fig. 5. The effect of surface superconductivity can be clearly observed as a large increase in $\sigma^{\prime}$ for $\ln (t *(H)) \leqslant-2$. For $\ln (t *(H))>-2$, the temperature dependence of $\sigma^{\prime}$ for $H \frac{f}{F} 0$ is very similar to the temperature dependence for $H=0$ and falls well below the temperature dependence predicted by the Maki theory (shown in the Fig. for $H=10 \mathrm{KG})$. It appears that in the limit where $t *(H) \rightarrow 0$ the theory and experiment will agree closely (for $H \neq 0$ ) although the existence of surface superconductivity prevents measurement of $\sigma^{\prime}$ in this region. In this limit the Maki theory predicts $\sigma^{\prime} \sim t \div(\mathrm{H})-\frac{1}{2}$ similar to the $\mathrm{A}-\mathrm{L}$ theory when $\mathrm{H}=0$. Finally, the magnitude of $\left(\sigma^{\prime} / \sigma_{0}\right)$ for fixed $t^{*}(H)$ is found to increase progressively with increasing field. The Maki theory does not account for this increase as the calculated magnitude of $\left(\sigma^{\prime} / \sigma_{0}\right)$ at constant $t^{*}(H)$ is nearly independent of $H$ when calculated based on eqn. (1). Also it should be mentioned that in the limit of $H \rightarrow 0$, the Maki expression differs from the A-L expression by a constant factor $(\sim 4)$. Since the two theories should agree both in magnitude and temperature dependence in this limit, it is concluded that 


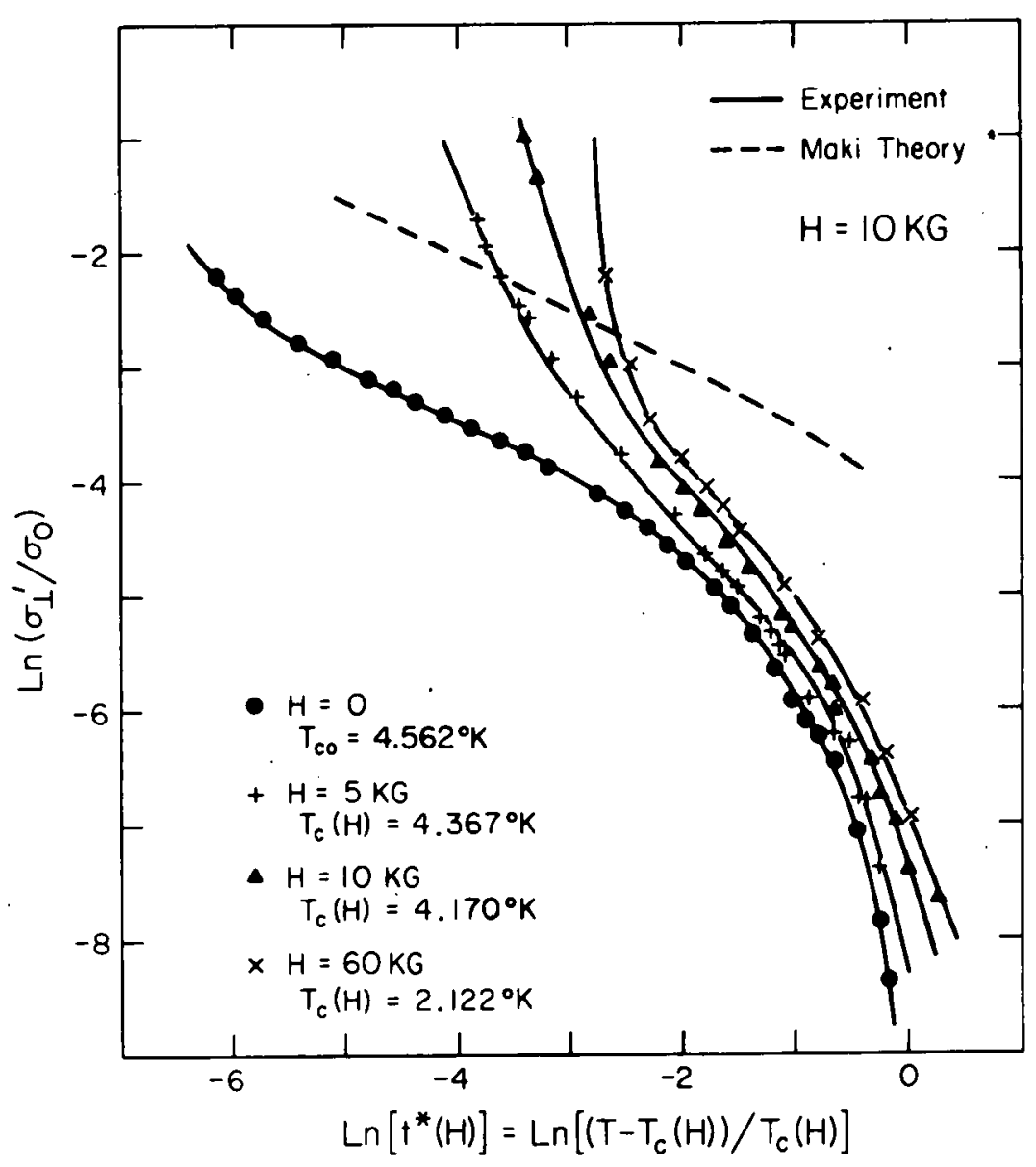

Fig. 5. The temperature dependence of the fluctuation conductivit y for amorphous $\mathrm{Zr}_{75} \mathrm{Rh}_{25}$ in constant applied magnetic field. The data are compared to the Maki theory illustrated in the figure for the case of $\mathrm{H}=10 \mathrm{KG}$. The $\mathrm{T}_{\mathrm{c}}$ of the sample in each applied field is given in the lower left hand corner. 
eqn. (1) may contain a numcrical error.

IV. BREAKDOWIN OF THEORY AT HIGH FIELD AND HIGH TEMPERATURE

As previously suggested in ref. 11, the breakdown of theory in the high temperature and high-field limit is most likely associated with the breakdown of the slow-variations approximation and the effect of nonlocal electrodynamical corrections to the Ginzburg-Landau theory. For the case of fluctuation diamagnetism, this problem has been considered in detail by several authors. Gollub et al. ${ }^{15}$ give a rather complete discussion of both the experimental and theoretical results pertaining to this problem. On the experimental side, they demonstrate a universal field dependence of $\left(M^{\prime} / \sqrt{H} T\right)$, where $M^{\prime}=$ excess diamagnetization, on the reduced variable $\mathrm{H}_{/ \mathrm{H}_{\mathbf{S}}}$. The field $\mathrm{H}_{\mathbf{S}}$ is a scaling field empirically determined for each metal and alloy studied. Empirically, $\mathrm{H}_{3}$ is defined as the field for which $\left(M^{\prime} / \sqrt{H} T\right)$ falls to one half the value predicted by the Ginzburg-Landau theory.

In the present study, we wish to consider the breakdown of the slow variations approximation as it effects both the field dependence and the temperature dependence of the paraconductivity. The effect of non-local electrodynamic corrections are ignored since, as discussed in ref. 15, these effects are small in the dirty limit. The amorphous superconductors represent the "extreme" dirty limit. A simple phenomenological modification of the Al theory is first considered which approximates the reduced role of short wavelength fluctuations and provides a good account of the observed temperature dependence of $\sigma^{\prime}$.

For a three dimensional superconductor, $A L^{6}$ express the super- 
current density for fluctuations as

$$
j_{\omega}=i \omega\left(\frac{\pi^{3}}{6}\right) c^{2} \frac{1}{m \rho_{0}} 2 \int_{0}^{\infty} \frac{q^{4} A \omega q}{\left(t *+\eta q^{2}\right)^{3}}
$$

where $C$ is a constant, $\rho_{0}$ the Fermi momentum, $A_{\omega}$ the vector potential, and $\eta$ reduces to the square of the zero temperature coherence length $\xi(0)$ in the dirty limit where the electron mean free path $\ell<\leqslant \xi_{0}$. Thompson ${ }^{20}$ first pointed out that for one and two dimensional superconductors, it is necessary to introduce a long wavelength cutoff in the $q$ integration to avoid unphysical singularities. Maki ${ }^{7}$, Patton ${ }^{9}$, and others have discussed the physical significance of the long wavelength cutoff which can be interpreted in terms of a pair-breaking parameter.

It is clear that a short wavelength cutoff must also apply to the fluctuation conductivity in one, two, or three dimensions. A lower bound for the cutoff wavevector is obtained by considering the following simple argument. Since roughly $10^{-3}$ of the total number of conduction electrons participate in superconductive pairing, in describing pairing phenomena one must consider at least the equivalent volume of $\sim 10^{3}$ atoms since such a volume is required to have a single pair. The characteristic dimension associated with this volume is $\sim 30 \AA$ and implies a short wavelength cutoff in any quantum mechanical description of a pair. The higher order correction terms in the Ginzburg-Landau formalism lead in fact to a more restrictive cutoff. For a dirty superconductor such terms are important for the case where $q \sim \bar{\zeta}(0)^{-1}$. The contributions to (3) for such $q$ vectors become important in considering the high temperature and high field limits of fluctuation phenomena. A detailed theory for the role of such $q$ fluctuations would 
require that one take into account the internal structure and dynamics of the bound pair. As a convenient means of phenomenologically treating the problem, we can simple introduce a short wavelength cutoff, $Q_{c}$ into the $q$ integration of equation (3) to approximated the reduced role of high q fluctuations.

We have experimentally observed a universal temperature dependence for $\sigma^{\prime}$. By using $Q_{C}$ as an adjustable parameter, one can attempt to fit the experimental data. Equation (3) was evaluated numerically as a function of $Q_{c}$ and the results used to fit the experimental temperature dependence of $\sigma^{\prime}$. The temperature dependence predicted by equation (3) was found to be rather sensitive to the choice of $Q_{c}$. It was found that the data are fit extremely well for $0.95 \lesssim 1.05$ where $Q_{c}$ is expressed in units of $\xi(0)^{-1}$. A typical set of curves used to fit the data for amorphous $\mathrm{Zr}_{75} \mathrm{Rh}_{25}$ are shown in Fig. 3. To within experimental error, it is clear that a $Q_{c} \approx g(0)^{-1}$ is required to described the data. This result is not surprising since the break down of the slow variations approximation is expected in just this range of $q$. A similar fit to the data for the La $80 \mathrm{Au}_{20}, \mathrm{Mo}_{30} \mathrm{Re}_{70}$, and $\mathrm{Nb}_{3} \mathrm{Ge}$ amorphous samples of ref. 11 gave a nearly identical result. We conclude that a cutoff wavector $Q_{C}=\xi(0)^{-1}$ provides an adequate mathematical description of the reduced role of high $q$ fluctuations as regards to the temperature dependence of $\sigma^{\prime}$.

An analysis of the field dependence of the fluctuation conductivity in terms of a cutoff wavector is more difficult. We can follow Gollub ${ }^{15}$ in attempting to describe the field dependence for $\sigma_{\perp}^{\prime}$ by determining the characteristic field for which significant deviations from theory occur. The data shown in Fig. 4 exhibit a very similar behavior as a function 
of reduced field $h$ as the dald of Fig. 3 as a function of reduced temperature. The natural scale for significant deviations in the field dependent behavior from theory is expected to be of similar magnitude to that found in the diamagnetic case. A simple argument ${ }^{21}$ which considers only the leading correction to the Ginsburg-Landau theory suggest that the slow variations approximation should break down for fields

$$
\mathrm{H} \sim \Phi_{\mathrm{o}} / 2 \pi \xi^{2}(0) \sim \mathrm{H}_{\mathrm{c} 2}(0)
$$

The characteristic field at which the experimental data depart from theory can be estimated by first noticing that the experimental curves of Fig. 4 can be brought into coincidence if the reduced field variable $h=\left(H-H_{c 2}(t)\right) / H_{c 2}(t)$ is redefined as $h *=\left(H-H_{c 2}(t) / H_{c 2}{ }^{(0) \text { which }}\right.$ amounts to measuring $\left(\mathrm{H}-\mathrm{H}_{\mathrm{c} 2}(\mathrm{t})\right)$ in units of $\mathrm{H}_{\mathrm{c} 2}(0)$. Comparison of data taken at different values of fixed $t$ shows that to within experimental error, all data are described rather well by a single universal curve when $\ell n\left(\sigma^{\prime} / \sigma_{0}\right)$ is plotted against $\ell n h^{*}$. One can then define a char acteristic value of $h *$ for which the data deviate from theory. The Maki theory predicts that $\sigma^{\prime}$ behaves like $\left[\mathrm{H}-\mathrm{H}_{\mathrm{c} 2}{ }^{(\mathrm{t})}\right]^{-\frac{1}{2}}$ for $\mathrm{H}>\mathrm{H}_{\mathrm{c} 2}{ }^{(\mathrm{t})}$ and $\left(\mathrm{H}-\mathrm{H}_{\mathrm{c} 2}(\mathrm{t})\right)$ small. We can estimate the deviation from the theory by determining the behavior of the slopes of the $\ell n\left(\sigma^{\prime} / \sigma_{0}\right)$ vs. $\ell n(h \%)$ curve $\left(s=d\left(\ln \sigma^{\prime}\right) / d \ell n(h *)\right)$ as a function of $h *$. For $h * \rightarrow 0$, the data approach $s=-\frac{1}{2}$ as predicted by theory. If we estimate the value of $h *$ for which $s$ deviates from $\frac{1}{2}$ significantly, we find for example that $s \approx 1$ for $h * \approx 0.15$ and $s \approx 2$ for $h * \approx 0.30$ (where $H_{c 2}(0)$ was taken to be the Maki extrapolated value in Fig. 1). Thus, significant devia- 
tions from theory nccur for lields of order of a few tenths of $\mathrm{H}_{\mathrm{c} 2}(0)$. The general features of the field dependence of $\sigma^{\prime}$ in the amorphous La ${ }_{78} \mathrm{Au}_{22}$ alloy are similar to those just discussed. It is concluded that the observed dependence of $\sigma^{\prime}$ on $h$ and the characteristic value of $h *$ for breakdown of the theory are general features of three dimensional amorphous superconductors.

In view of the present data, it would seem to be worthwhile to extend the theoretical results of AL, Maki, Patton and those mentioned in ref. 15 for the case of the diamagnetic fluctuations to the case of fluctuation conductivity in high field and at high temperature. It seems likely that a suitable extension of the microscopic theory can provide a complete and consistent picture for understanding the experimental data. Such an extension would provide a detailed test of the microscopic well beyond the limits of validity of the approximations made in the Ginzburg-Landau theory.

\section{SUMMARY}

Measurements of the paraconcuctivity $\sigma^{\prime}$ as a function of temperature and applied magnetic field for a three dimensional amorphous superconductor have been presented. The data have been compared with several current theories. For temperatures close to $T_{c}$ and small fields, the dependence of $\sigma^{\prime}$ on $T$ and $H$ is well described by the theories of Aslamasov and Larkin, Maki, and others. For high temperature and large fields, the experimental data give values of $\sigma$ ' which fall well below those predicted by theory. This discrepancy is attributed to a break down of the slow variations approximation which is 
implicitly made in both the Ginzburg-Landau theory of fluctuations and in the current ulicroscopıc theories. A convenient phenomenological description of the temperature dependence of $\sigma^{\prime}$ is obtained by assuming a short wavelength cutoff in the A-L theory in order to account for the reduced role of high $q$ fluctuations. A good fit to the data is obtained by taking this cutoff wavevector to be $Q_{c} \approx \xi(0)^{-1}$. A complete theory must take into account the higher order terms in the Ginzburg-Landau expansion of the free energy and in so doing account for the internal structure of a bound pair. It is hoped that the present work will stimulate theoretical interest in appropriately extending the theory of paraconductivity to account for the above mentioned discrepancies. It is noteworthy that such an extension has already been considered for the case of describing diamagnetic fluctuations. 


\section{REFERENCES}

1. R. E. Glover, III, Phys. Letters 25A, 542 (1967) D. C. Naugle and R. E. Glover, III, Phys. Letters 28A, 110 (1968).

2. J. E. Crow, A. K. Bhatnagar, and T. Mihalisin, Phys. Rev. Letters 28,25 (1972).

3. W. E. Masker and R. D. Parks, Phys. Rev. B1, 2164 (1970).

4. R. A. Craven, G. A. Thomas, and R. D. Parks, Phys. Rev. B , 157 (1973).

5. J. W. Lue, A. G. Montgomery, and R. R. Hake, Phys. Rev. B 11, $3393(1975)$

6. L. G. Aslamazov and A. I. Larkin, Phys. Letters A 26, 238 (1968). L. G. Aslamazov and A. I. Larkin, Sov. Phys. Solid State, 10, 875 (1968).

7. a) K. Maki, Prog. Theor. Phys. 39, 897 (1968).

b) K. Maki, Prog. Theor. Phys. 40, 193 (1968).

c) K. Maki, J. of Low Temp. Phys. 1, 513 (1969).

8. K. D. Usadel, Z. Physik 227, 260 (1969).

9. B. R. Patton, Phys. Rev. Letters 27, 12.73 (1971).

10. B. Keck and A. Schmid, Solid State Commun. 17, 799 (1975).

11. W. L. Johnison and C. C. Tsuei, Phys. Rev. B 13, 4827 (1976). 
12. H. Schmidt; Z. Physik 216, 336 (1968).

13. R. E. Prange, Phys. Rev. B1, 2349 (1970).

14. B. R. Patton, V. Ambegaokar, and J. W. Wilkins, Solid State Commun. ?, 1287 (1969).

15. J. P. Gollub, M. R. Beasley and M. Tinkham, Phys. Rev. Letters 25, 1646 (1970).

J. P. Gollub, M. R. Beasly, R. Callarotti, and M. Tinkham, Phys. Rev. 7, 3039 (1973).

16. P. Duwez, Progress in Solid State Chemistry, (P ermagon Press, Oxford and New York) 1966.

17. K. Tagano and K. Tachikawa, J. of Applied Phys. 46, 3609 (1975).

18. K. Maki, Physics (N. Y.) 1, 127 (1964).

19. Richard S. Thompson, Phys. Rev. B, 1, 327 (1970).

20. N. R. Werthamer, Superconductivity, edited by R. D. Parks (Marcel Decker, New York, 1969) p. 321. 